\title{
Static Headspace Extraction with Compounds Trapping for the Analysis of Volatile Lipid Oxidation Products
}

\author{
Henryk Jeleń $^{1}$ - Anna Gracka ${ }^{1}$ - Barbara Myśków ${ }^{1}$
}

Received: 28 November 2016/Accepted: 31 January 2017 /Published online: 12 February 2017

(C) The Author(s) 2017. This article is published with open access at Springerlink.com

\begin{abstract}
Static headspace is an easy to perform, convenient, and usually automated method for extraction of volatiles from various food matrices. Among other applications, it is used also for monitoring of volatile lipid oxidation products. However, the biggest disadvantage of this method is its low sensitivity especially when compared to such techniques as solid-phase microextraction or dynamic headspace. This paper focuses on the comparison of static headspace analysis of volatile lipid oxidation products with a modification of this technique, where volatiles extracted multiple times from oil matrix in a headspace vial are trapped on Tenax trap and subsequently desorbed into gas chromatograph. Twenty volatile compounds (mainly alkanals, 2-alkenals, ketones, and alcohols being volatile lipid oxidation products) in rapeseed oil were used for method comparison. For all compounds, a significant increase in method sensitivity and improvement of limits of detection (10 to 33 times) depending on the compound were noted; however, the repeatability was worse (for 16 out of 20 compounds) and carry-over values (also 16/20) were higher for combined headspace/trapping method.
\end{abstract}

Keywords Rancidity · Lipid oxidation products · Volatiles · Aldehydes $\cdot$ Headspace $\cdot$ Tenax $\cdot$ Rapeseed oil

Henryk Jeleń

henrykj@up.poznan.pl

1 Faculty of Food Science and Nutrition, Poznań University of Life Sciences, Wojska Polskiego 31, 60-624 Poznań, Poland

\section{Introduction}

Aldehydes are volatile compounds which may indicate the deterioration of food, especially of fat rich foods as a result of processing, storage, or seed pre-treatment. These compounds are the main products of the oxidation of unsaturated fatty acids (Mandić et al. 2013). Hexanal, one of the most common aldehydes in food, was explored in several studies as the indicator of oxidation in oils (Azarbad and Jelen 2014); however, the hydroperoxides decomposition in the process of autooxidation results in formation of many other aldehydes (alkanals, 2-alkenals, 2,4-alkadienals as the main ones), and also ketones, alcohols, and furanes, to name the most prevailing groups of compounds.

Volatile compounds, among them, volatile lipid oxidation product analysis is a challenging task because of their physical properties, food matrices they are released from and often their trace levels in food. The use of solvents allows for exhaustive extraction of volatile compounds; on the other hand, organic solvents may dilute or mask analytes during GC analysis (Guth and Grosch 1993). Employment of high temperature for the isolation of volatile compounds using distillation methods may result in their degradation, or in formation of artifacts. Headspace techniques such as static or dynamic headspace (SHS or DHS, respectively), headspace sorptive extraction (HSSE), solid-phase microextraction (SPME), and direct thermal desorption (DTD) are robust methods which do not require the use of a solvent or circumstances which impede obtaining a pure volatile compound extract of the analyzed sample (Bylaite and Meyer 2006; Cavalli et al. 2003). Each of these methods enables the isolation of volatile compounds usually without artifacts' formation. Purge and trap (PT) method is used for volatile compounds' isolation but, despite its high capacity, may adsorb water and cause artifacts formation during analysis (Manzini et al. 2011). HSSE and SPME are solvent-free methods based on various stationary 
phases. SPME invented in the beginning of 90-ties (Arthur and Pawliszyn 1990) became quickly one of most often sample preparation method used for volatile and semi-volatile compounds. Its simplicity of use and high range of available sorbents makes it a useful tool for volatiles and flavor compounds analysis in food (Jeleń et al. 2012). Nowadays, SPME has been used in many studies into volatiles in oils (Benincasa et al. 2003; Cecchi and Alfei 2013; Wei et al. 2015). HSSE is used for extracting volatile compounds from gaseous and aqueous phases (known as stir-bar sorptive extraction, SBSE) with the use of a stir-bar, usually coated with adsorbents such as polydimethylsiloxane (PDMS) (Sánchez-Rojas et al. 2009). The static and dynamic headspace extraction techniques are simple and robust methods, but - compared with other solventless methods - SHS has lower sensitivity (Wenzl and Lankmayr 2002). Despite its relatively low sensitivity, SHS with mass spectrometer has been used with satisfying results for differentiation between olive oils or detecting adulteration in virgin olive oils (Lorenzo et al. 2002; Marcos Lorenzo et al. 2002). Recent review by Sghaier and coworkers summarize different approaches to analysis of plant oil volatile compounds (Sghaier et al. 2016). Combining simplicity and robustness of static headspace analysis in its automated form with trapping volatiles on polymers in a single sampling unit may be the solution to overcome low sensitivity issues of SHS.

The aim of the study was to compare the extraction of volatile lipid oxidation products (VLOP) from rapeseed oil using static headspace method, in which typical loop transfer for compound injection was used, with a combination of headspace method in which compounds can be extracted multiple times from a headspace vial into a Tenax trap before transferring them into gas chromatograph.

\section{Materials and Methods}

\section{Reagents and Standards}

The following compounds were used to represent different classes of VLOP: alkanals - pentanal, hexanal, octanal, nonanal, decanal; 2-alkenals-2-pentenal, 2-hexenal, 2-heptenal, 2nonenal; 2,4-alkadienals - 2,4-heptadienal and 2,4-decadienal; unsaturated alcohols -2-pentene-1-ol, 3-hexene-1-ol, 2-hexene1-ol, 1-octene-3-ol; ketones-1-pentene-3-one, 6-methyl-5hepten-2-one, 2-pentanone, 2-heptanone; and esters-3-hexenyl acetate, 3-hexyl acetate and also 2-pentylfurane. All standards were purchased at Sigma-Aldrich (Poznań, Poland) in the highest available purity (usually $98-99 \%$, GC).

\section{Sample Preparation and GC/MS Analysis}

For method development, a mixture of volatile lipid oxidation products (VLOP) was prepared in refined deodorized rapeseed oil. Standards were prepared gravimetrically. To obtain working solutions, the stock solution was dissolved in fresh refined rapeseed oil to obtain $1 \mathrm{mg} / \mathrm{L}$ solution, which was used for subsequent analyses, when method parameters were evaluated and appropriate solutions were prepared for linearity and LOD testing.

Analyses were performed on GC/MS-TQ8030 (Shimadzu, Japan) triple quadrupole mass spectrometer system. The gas chromatograph (GC-2010 Plus) was equipped with a single split/splitless injection port and a Zebron ZB-5 column (30 $\mathrm{m} \times 0.25 \mathrm{~mm} \times 0.25 \mu \mathrm{m}$, Phenomenex). The GC/MS system was equipped with AOC-20i autoinjector. The GC/ MS system was used in a single quadrupole mode with the first quadrupole set to transmission of all ions. All headspace samples were introduced into GC/MS by direct interface, using HS-20 Trap (Shimadzu, Japan) static headspace autosampler with adsorbent trap. The absorbent trap dimension was $2 \mathrm{~mm}$ (ID) $\times 100 \mathrm{~mm}$ and the trap was filled with Tenax TA (amount not specified). Parameters for the extraction of volatiles using HS-20 Trap were as follows: for injections in "loop" mode (static headspace) - sample line temp. $140{ }^{\circ} \mathrm{C}$; transfer line $140{ }^{\circ} \mathrm{C}$; pressure equilibrium time $0.1 \mathrm{~min}$; load time $0.5 \mathrm{~min}$; load equilibrium $0.1 \mathrm{~min}$; injection time $0.5 \mathrm{~min}$; GC cycle time 40 min. For "trap" mode, additionally, trap parameters were set as follows: trap cooling temp. $-25^{\circ} \mathrm{C}$; trap equilibrium temp. $-25^{\circ} \mathrm{C}$. Trap desorption temp. varied depending on the experiment (tested in a 200 $300{ }^{\circ} \mathrm{C}$ range). Multi injection count for trap varied (1-10, depending on the experiment). For both modes, the following parameters were evaluated in method development: HS-20 Trap oven temperature, shaking level (1-5), equilibrium time (10-60 $\mathrm{min})$, and injection time (0.5 and $1 \mathrm{~min})$.

\section{Results and Discussion}

To develop and efficient extraction method using static headspace analysis basic parameters, such as extraction temperature, incubation time, shaking intensity, and injection parameters, were optimized to achieve maximal sensitivity. Static headspace autosampler used in these experiments used injection by loop, so the volatile compounds after equilibrium was established were transferred to the thermostated loop, from which they were swept by the carrier gas into gas chromatograph. Basic extraction parameters tested are shown in Fig. 1. In the graph, total peak areas of standards used in method elaboration are shown. Temperatures ranging from 40 to $90{ }^{\circ} \mathrm{C}$ were evaluated and the threefold increase was obtained when extraction at the lowest and the highest temperatures were compared. Because of the instability of the matrix and some standards, especially in regard to possible oxidation processes, for further experiments, extraction temperature of $70{ }^{\circ} \mathrm{C}$ was chosen. For this temperature, extraction time 

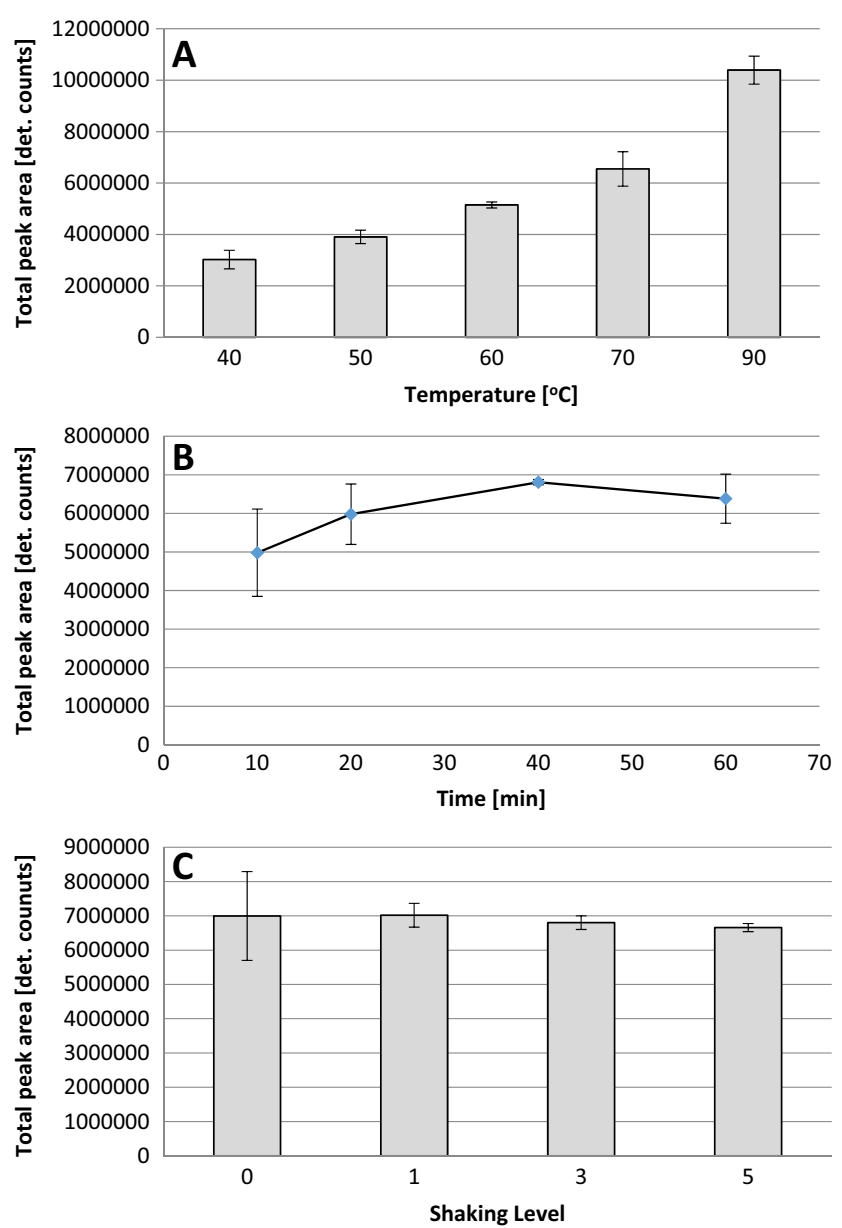

Fig. 1 Development of optimal parameters for HS extraction of VLOP using loop system. a Influence of temperature on the extraction of volatile compounds from oil (extraction time $30 \mathrm{~min}$ ). b Influence of extraction time on the extraction of volatile compounds from oil (extraction temperature $90^{\circ} \mathrm{C}$ ). $\mathbf{c}$ Influence of shaking intensity on the extraction of volatile compounds from oil

ranging from 10 to 60 min was tested. As shown in Fig. 1b, the increase was noted from 10 to 40-min extraction and the reproducibility was the highest at 40-min extraction, which was used for further analyses. Interestingly, the repeatability was the lowest at the shortest, but also the longest extraction times. As the migration of volatiles from the matrix is influenced by mixing, to facilitate fasted transfer to the headspace, shaking levels (alternative of mixing speed in autosampler) were tested. With the highest degree of shaking, the repeatability of peak areas was the highest. Therefore, the highest shaking level was used for subsequent analyses. Additionally, different combinations of pressurizing time and injection time were compared, to optimize injection process (results not shown). The highest total peak areas were obtained for pressurizing time $1 \mathrm{~min}$ and injection time $0.5 \mathrm{~min}$; however, the values differed for particular compounds, especially with regard to pressurizing time.

The headspace autosampler was equipped with a Tenax A filled trap that can be cooled and multiple extractions can be performed from a single vial one, after one, without achieving equilibrium, which differs this method from multiple headspace extraction (MHE). Cooling the trap improves adsorption on the polymer and up to ten extractions can be performed for a single sample. Figure 2 illustrates the increase in peak areas of compounds when different extraction numbers were applied. In Fig. 2a, total peak areas are shown, whereas in Fig. 2b, peak areas of (E)-2-nonenal are presented as an example. Dramatic increase in peak areas can be noted when one extraction is compared to ten extractions. However, the high number of extractions despite trap cooling and high desorption temperatures may result in column overloading and decrease in peak symmetry and quality. Manipulating with split ratio improves peak shapes, but results in lower sensitivity, so the balance between these two factors has to be elaborated depending on the type of sample and compounds analyzed. For our set of compounds, the optimal number of extractions that did not degrade significantly chromatographic separation was seven, and it was used for method elaboration. Higher number of extractions ended in serious peak overlapping. For all extractions using trap system, trap temperature was kept at $-25{ }^{\circ} \mathrm{C}$ and different desorption temperatures were tested $\left(200,250,275\right.$, and $\left.300{ }^{\circ} \mathrm{C}\right)$. The highest total peak area was observed for desorption temperature of 275 and $300{ }^{\circ} \mathrm{C}$ (detailed results not shown). The peak areas of desorbed compounds at different temperatures differed substantially. For example, hexanal peak area after desorption at $300{ }^{\circ} \mathrm{C}$ was $133 \%$ of that at temperature of $200{ }^{\circ} \mathrm{C}$, but the peak area of 2,4-decadienal desorbed at $300^{\circ} \mathrm{C}$ was $433 \%$ of
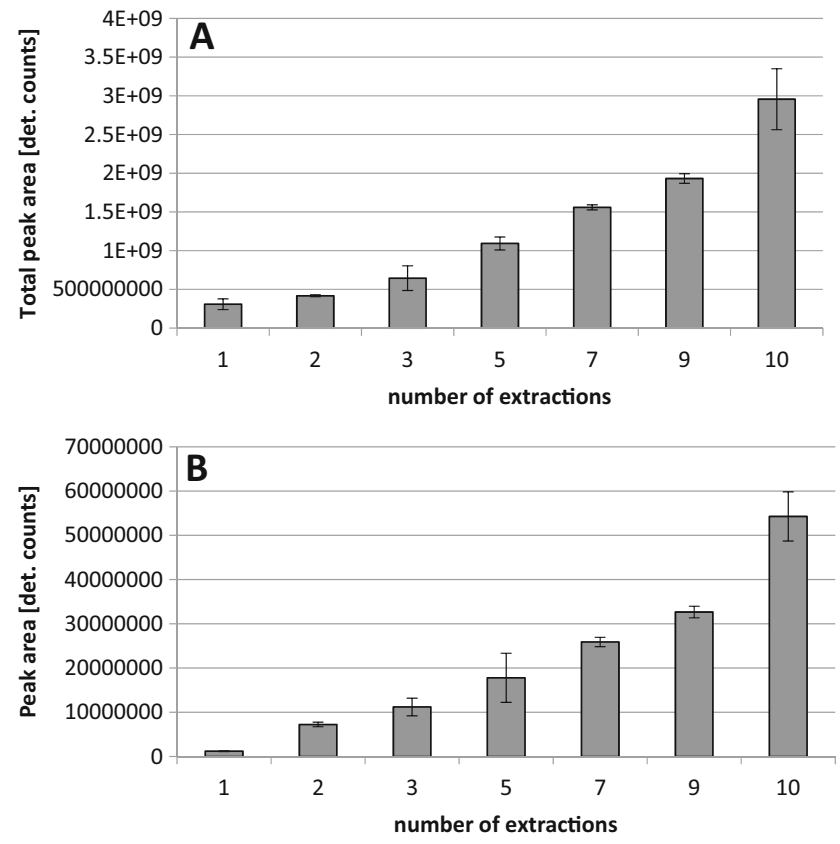

Fig. 2 Changes in peak areas as a result of number of extraction performer using headspace sampler in a "trap" mode. a Total peak area for all compounds used for method development. b Peak areas of (E)-2nonenal 
the peak area of that compound desorbed at $200{ }^{\circ} \mathrm{C}$. The carryover for 2,4-decadienal was the highest among all tested compounds. To minimize carry-over desorption temperature of $300{ }^{\circ} \mathrm{C}$ was chosen for the analyses. Table 1 shows basic performance parameters for both methods. For "loop" injection mode, both the reproducibility and carry-over are higher than for "trap" method for majority (16/20) compounds. Linearity of both methods which were tested in a range of $0.1-20$ or $0.1-50 \mathrm{mg} / \mathrm{L}$ was comparable. The highest difference was noted in the LOQ values and sensitivity of both methods. Gain in sensitivity can be seen in Fig. 3, where standard curves for hexanal and (E)-2-nonenal are shown. Dramatic differences indicate the potential of trapping volatiles in the headspace for determination of these volatiles. Similar differences were noted for the remaining compounds. When differences between LOQ for "loop" and "trap" methods are compared (Table 1), the latter one provides from 10 to 33 times lower LOQs depending on a compound. The limits of quantitation for lipid oxidation products are very low, regarding their presence in oils, and can compete with LOQs achieved using SPME. A potential advantage of "trap" method is the high linear range, which was for the majority of compounds in a $0.1-20 \mathrm{mg} / \mathrm{L}$ range, whereas in SPME sampling usually the linear range is often limited, especially when fibers based on polymers are used (carboxene/divinylbenzene or PDMS, carboxene/PDMS), which are very often selected to obtain low limits of detection. Linear range for "trap" method was lower than "loop" for majority of compounds (for 12 out of 20 the linear range was $0.1-20 \mathrm{mg} / \mathrm{L}$ ), but this was related to adsorption of the analytes on limited sites (pores) of Tenax surface. Nevertheless, the Tenax surface is much higher than for microextraction methods; therefore, the linear range was satisfactory.

Static headspace analysis of volatile compounds was used as a method for monitoring oxidative stability of various oils in which total volatiles peak areas were measured (Warner et al. 1989). Automated static headspace is a common extraction technique in oil production plants, where it is used for both the residue extraction solvent analysis and volatile compounds in oils. In comparative studies on volatile compounds in oils, static headspace, along with dynamic headspace and direct injection, was used when inter-laboratory collaborative studies were performed to monitor, among other parameters, pentane and hexanal in oils (Warner and Nelsen 1996).

Table 1 Basic characteristics for methods using static headspace extraction (loop) and static headspace extraction with compounds trapping on Tenax trap (trap)

\begin{tabular}{|c|c|c|c|c|c|c|c|c|c|c|c|}
\hline \multirow[t]{2}{*}{ Compound } & \multirow[b]{2}{*}{$\begin{array}{l}\text { Rt } \\
{[\mathrm{min}]}\end{array}$} & \multicolumn{2}{|c|}{ Reproducibility } & \multicolumn{2}{|c|}{ Carry-over } & \multicolumn{2}{|c|}{ Linearity } & \multirow[b]{2}{*}{$\begin{array}{l}\text { Range } \\
\mathrm{mg} / \mathrm{L}\end{array}$} & \multicolumn{2}{|l|}{ LOQ } & \multirow[b]{2}{*}{ GAIN } \\
\hline & & $\begin{array}{l}\text { Loop } \\
\text { RSD [\%] }\end{array}$ & Trap & $\begin{array}{l}\text { Loop } \\
{[\%]}\end{array}$ & Trap & $\begin{array}{l}\text { Loop } \\
R^{2}\end{array}$ & Trap & & $\begin{array}{l}\text { Loop } \\
\mathrm{mg} / \mathrm{L}\end{array}$ & Trap & \\
\hline 1-penten-3-one & 2.793 & 9.53 & 4.01 & 0.64 & 0.39 & 0.994 & $0.991 *$ & $0.1-20$ & 0.1 & 0.009 & 11 \\
\hline pentanal & 2.936 & 6.93 & 4.53 & 1.06 & 0.26 & 0.994 & $0.980 *$ & $0.1-20$ & 0.1 & 0.005 & 21 \\
\hline 2-pentenal, (E)- & 3.654 & 6.69 & 7.78 & 0.48 & 4.84 & 0.999 & $0.991 *$ & $0.1-50$ & 0.1 & 0.006 & 16 \\
\hline 2-penten-1-ol, (Z)- & 3.862 & 5.63 & 7.23 & 0.5 & 1.16 & 0.999 & $0.996^{*}$ & $0.1-50$ & 0.1 & 0.006 & 17 \\
\hline Hexanal & 4.353 & 4.49 & 7.56 & 2.57 & 6.92 & 0.999 & 0.999 & $0.1-50$ & 0.1 & 0.005 & 22 \\
\hline 2-hexenal, (E)- & 5.282 & 3.44 & 8.73 & 0.32 & 2.71 & 0.997 & $0.986^{*}$ & $0.1-50$ & 0.1 & 0.007 & 15 \\
\hline 2-hexen-1-ol, (Z)- & 5.502 & 1.49 & 5.32 & 0.51 & 3.99 & 0.998 & $0.995 *$ & $0.1-50$ & 0.1 & 0.006 & 18 \\
\hline 2-heptanone & 5.960 & 7.48 & 3.82 & 0.61 & 0.74 & 0.999 & $0.991 *$ & $0.1-50$ & 0.05 & 0.004 & 13 \\
\hline Heptanal & 6.210 & 5.52 & 16.36 & 2.72 & 11.78 & 0.983 & $0.981 *$ & $0.2-50$ & 0.2 & 0.020 & 10 \\
\hline 2-heptenal, (Z)- & 7.306 & 5.47 & 4.95 & 0.46 & 2.89 & 0.999 & 0.995 & $0.1-50$ & 0.1 & 0.005 & 20 \\
\hline 1-octen-3-ol & 7.770 & 3.29 & 4.74 & 1.40 & 10.36 & 0.994 & 0.998 & $0.1-20$ & 0.1 & 0.003 & 33 \\
\hline 5-hepten-2-one, 6-methyl- & 7.861 & 3.90 & 5.56 & 0.74 & 1.35 & 0.999 & 0.997 & $0.1-50$ & 0.1 & 0.005 & 19 \\
\hline Furan, 2-pentyl- & 7.995 & 4.77 & 6.02 & 0.56 & 2.27 & 0.999 & $0.993 *$ & $0.1-50$ & 0.1 & 0.006 & 17 \\
\hline 2,4-heptadienal, (E,E)- & 8.120 & 1.56 & 6.62 & 2.29 & 0.51 & 0.913 & $0.995^{*}$ & $0.3-20$ & 0.3 & 0.020 & 15 \\
\hline 3-hexen-1-ol, acetate, (E)- & 8.265 & 4.70 & 5.45 & 1.99 & 3.05 & 0.999 & $0.992 *$ & $0.1-50$ & 0.04 & 0.002 & 17 \\
\hline 2-nonanone & 9.998 & 4.56 & 10.84 & 2.15 & 1.13 & 0.999 & $0.994 *$ & $0.1-50$ & 0.1 & 0.006 & 18 \\
\hline Nonanal & 10.287 & 4.91 & 11.96 & 0.88 & 14.09 & 0.998 & 0.999 & $0.1-50$ & 0.03 & 0.002 & 20 \\
\hline 2-nonenal, (E)- & 11.360 & 5.18 & 5.91 & 0.57 & 8.59 & 0.999 & 0.999 & $0.1-50$ & 0.1 & 0.005 & 19 \\
\hline Decanal & 12.234 & 3.30 & 10.01 & 7.00 & 8.12 & 0.994 & 0.998 & $0.1-50$ & 0.1 & 0.005 & 21 \\
\hline 2,4-decadienal, (E,E)- & 13.898 & 7.47 & 8.04 & 5.84 & 10.52 & 0.792 & 0.996 & $0.2-20$ & 0.5 & 0.019 & 27 \\
\hline
\end{tabular}

Linearity $R^{2}$ values with asterisk $(*)$ indicate that for TRAP method range was $0.1-20 \mathrm{mg} / \mathrm{L}$

$R t$ retention time, RSD relative standard deviation, $L O Q$ limit of quantitation, GAIN limit of quantitation decrease when trap and loop methods are compared 

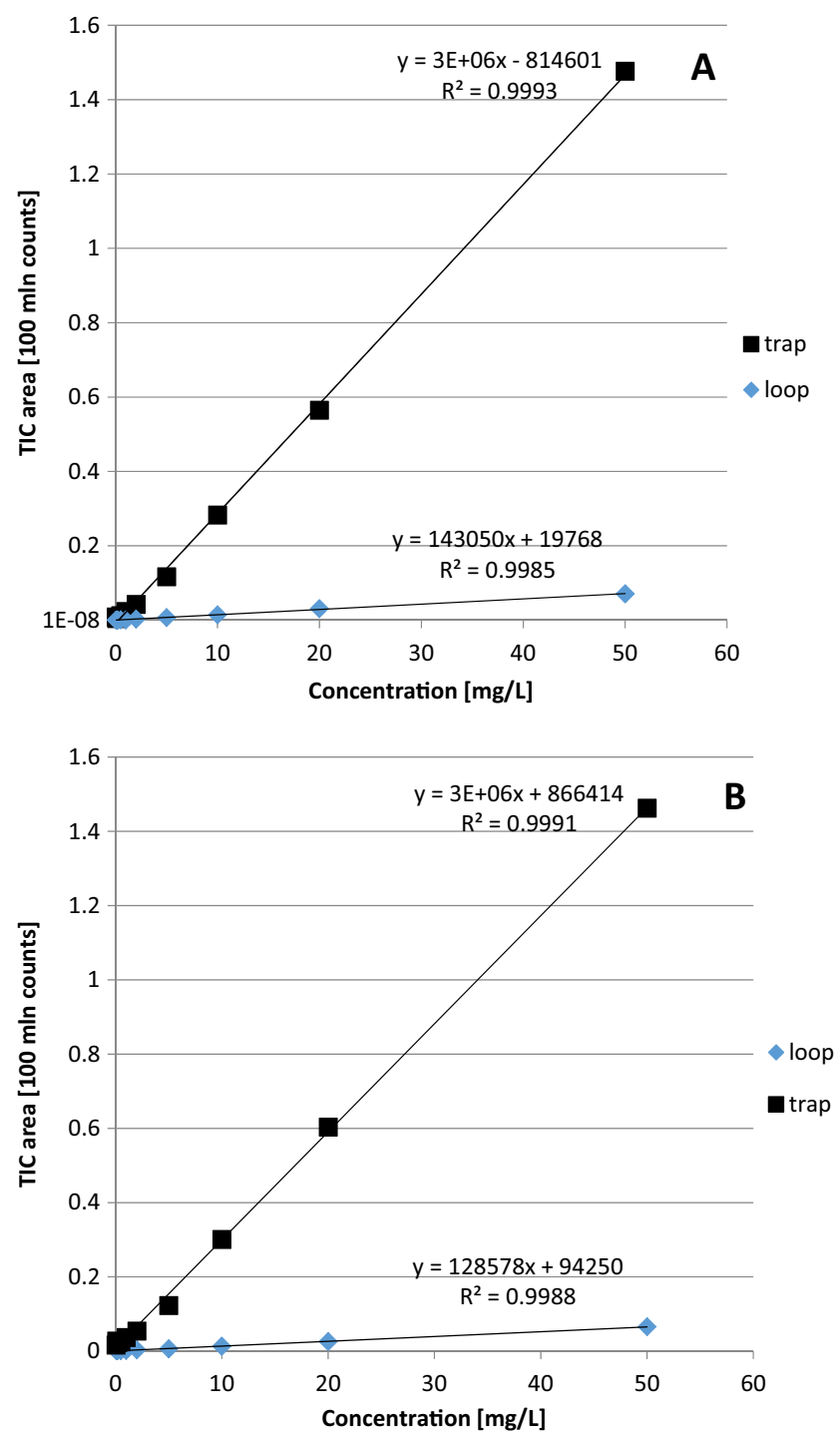

Fig. 3 Calibration curves for static headspace using "loop" and "trap" approaches (with seven extractions from a single vial) for two aldehydes. a Hexanal. b (E)-2-nonenal

Authors noted lower area counts for static headspace compared to dynamic headspace methods, but stated that static headspace can be a method capable of determination of oxidation level. Snyder et al. 1988 compared static headspace and purge and trap noting the much higher proportions of 2,4-decadienal and 2,4-heptadienal in trapping technique compared to static headspace. However, the static headspace produced higher proportions of low molecular compounds. Indeed, the advantage of static headspace technique is its high sensitivity for low-boiling compounds. This is due to the relation of vapor pressure of compounds in the homolog series to the number of carbon atoms in the molecule. The use of SPME for volatile compounds of plant oils has been verified in many works and it proved to be a sensitive method by which tens of compounds could be monitored in fresh and oxidized oils. Limits of detection in low micrograms per liter can be achieved (Jeleń et al. 2000). SPME has been proposed as a method for analysis of potential markers for oxidation status (nonanal proposed as indicator) (Vichi et al. 2003), or quality - freshness markers of olive oil ((E)-hex-2enal, C6 alcohols and C5 ketones) (Cavalli et al. 2004), although in many papers describing its use in flavor analysis, it is based mainly on area count comparison, not a quantitative method (Jeleń et al. 2012). A comparative study was performed in which static headspace was compared to headspace solid-phase microextraction, headspace sorptive extraction and direct thermal desorption (Cavalli et al. 2003). Compared to sorptive methods, static headspace showed very low sensitivity and was concluded to be not suitable for characterization of olive oil volatile compounds for that reason.

Presented in this paper, combination of headspace with compounds trapping can dramatically increase the sensitivity and therefore contribute to its use also for low levels of volatile compounds to be quantified.

\section{Conclusions}

Presented comparison shows that combining static automated headspace analysis, where a certain volume of headspace is transferred to the gas chromatograph without sample enrichment, with multiple extraction and trapping volatiles before their injection results in significant improvement of method sensitivity. As the trapping system is located in the automated static headspace sampler, it provides a relatively simple from technical point of view and serious from method performance point of view enhancement of its analytical abilities. Combining static headspace with compounds trapping overcomes the most severe static headspace drawback-low sensitivity, maintaining high linearity and its robustness and sample preparation simplicity. To minimize the risk of column overloading and carry-over issues, the method needs compromise in number of extractions and desorption temperatures, which depend highly on the analyte character and the matrix.

\section{Compliance with Ethical Standards}

Funding This study was funded by the National Science Centre, Poland (grant number 2012/07/N/NZ9/01634).

Conflict of Interest Henryk Jelen declares that he has no conflict of interest. Anna Gracka declares that she has no conflict of interest. Barbara Myśków declares that she has no conflict of interest.

Ethical Approval This article does not contain any studies with human participants or animals performed by any of the authors.

Informed Consent Not applicable. 
Open Access This article is distributed under the terms of the Creative Commons Attribution 4.0 International License (http:// creativecommons.org/licenses/by/4.0/), which permits unrestricted use, distribution, and reproduction in any medium, provided you give appropriate credit to the original author(s) and the source, provide a link to the Creative Commons license, and indicate if changes were made.

\section{References}

Arthur CL, Pawliszyn J (1990) Solid phase microextraction with thermal desorption using fused silica optical fibers. Anal Chem 62(19): 2145-2148

Azarbad MH, Jelen H (2014) Determination of hexanal — an indicator of lipid oxidation by static headspace gas chromatography (SHS-GC) in fat-rich food matrices. Food Anal Methods 8(7):1727-1733

Benincasa C, De Nino A, Lombardo N, Perri E, Sindona G, Tagarelli A (2003) Assay of aroma active components of virgin olive oils from southern Italian regions by SPME-GC/ion trap mass spectrometry. J Agric Food Chem 51(3):733-741

Bylaite E, Meyer AS (2006) Characterisation of volatile aroma compounds of orange juices by three dynamic and static headspace gas chromatography techniques. Eur Food Res Technol 222(1-2):176184

Cavalli J-F, Fernandez X, Lizzani-Cuvelier L, Loiseau A-M (2003) Comparison of static headspace, headspace solid phase microextraction, headspace sorptive extraction, and direct thermal desorption techniques on chemical composition of French olive oils. J Agric Food Chem 51(26):7709-7716

Cavalli J-F, Fernandez X, Lizzani-Cuvelier L, Loiseau A-M (2004) Characterization of volatile compounds of French and Spanish virgin olive oils by HS-SPME: identification of quality-freshness markers. Food Chem 88:151-157

Cecchi T, Alfei B (2013) Volatile profiles of Italian monovarietal extra virgin olive oils via HS-SPME-GC-MS: newly identified compounds, flavors molecular markers, and terpenic profile. Food Chem 141(3):2025-2035

Guth H, Grosch W (1993) Identification of potent odourants in static headspace samples of green and black tea powders on the basis of aroma extract dilution analysis (AEDA). Flavor Fragrance J 8:173178

Jeleń HH, Obuchowska M, Zawirska-Wojtasiak R, Wąsowicz E (2000) Headspace solid phase microextraction use for the characterization of volatile compounds in vegetable oils of different sensory quality. $\mathrm{J}$ Agric Food Chem 48:2360-2367
Jeleń HH, Majcher M, Dziadas M (2012) Microextraction techniques in the analysis of food flavor compounds: a review. Anal Chim Acta 738:13-26

Lorenzo IM, Pavón JLP, Laespada MEF, Pinto CG, Cordero BM, Henriques LR, Lopez PS (2002) Application of headspace-mass spectrometry for differentiating sources of olive oil. Anal Bioanal Chem 374(7-8): 1205-1211

Mandić AI, Sedej IJ, Sakač MB, Mišan AČ (2013) Static headspace gas chromatographic method for aldehyde determination in crackers. Food Anal Methods 6(1):61-68

Manzini S, Durante C, Baschieri C, Cocchi M, Sighinolfi S, Totaro S, Marchetti A (2011) Optimization of a dynamic headspace - thermal desorption - gas chromatography/mass spectrometry procedure for the determination of furfurals in vinegars. Talanta 85(2):863-869

Marcos Lorenzo I, Pérez Pavón JL, Fernández Laespada ME, García Pinto C, Moreno Cordero B (2002) Detection of adulterants in olive oil by headspace-mass spectrometry. J Chromatogr A 945(1-2): 221-230

Sánchez-Rojas F, Bosch-Ojeda C, Cano-Pavón JM (2009) A review of stir bar sorptive extraction. Chromatographia 69(S1):79-94

Sghaier L, Vial J, Sassiat P, Thiebaut D, Watiez M, Breton S, Rutledge DN, Cordella CBY (2016) An overview of recent developments in volatile compounds analysis from edible oils: technique-oriented perspectives. Eur J Lipid Sci Technol. doi:10.1002/ejlt.201500508

Snyder JM, Frankel EN, Selke E, Warner K (1988) Comparison of gas chromatographic methods for volatile lipid oxidation compounds in soybean oil. JAOCS 65(10):1617-1620

Vichi S, Pizzale L, Conte SF, Buxaderas S, López-Tamames E (2003) Solid phase microextraction in the analysis of virgin olive oil volatile fraction: modifications induced by oxidation and suitable markers of oxidative status. J Agric Food Chem 51:6564-6571

Warner K, Frankel EN, Mounts TL (1989) Flavor and oxidative stability of soybean, sunflower and low erucic acid rapeseed oils. JAOCS 66(4):558-564

Warner K, Nelsen T (1996) AOCS collaborative study on sensory and volatile compounds analyses of vegetable oils. JAOCS 73(2):157166

Wei CQ, Liu WY, Xi WP, Cao D, Zhang HJ, Ding M, Huang KX (2015) Comparison of volatile compounds of hot-pressed, cold-pressed and solvent-extracted flaxseed oils analyzed by SPME-GC/MS combined with electronic nose: major volatiles can be used as markers to distinguish differently processed oils: volatile compounds of different processed flaxseed oils. Eur J Lipid Sci Technol 117(3):320 330

Wenzl T, Lankmayr E (2002) Comparative studies of the static and dynamic headspace extraction of saturated short chain aldehydes from cellulose-based packaging materials. Anal Bioanal Chem 372(5-6): 649-653 